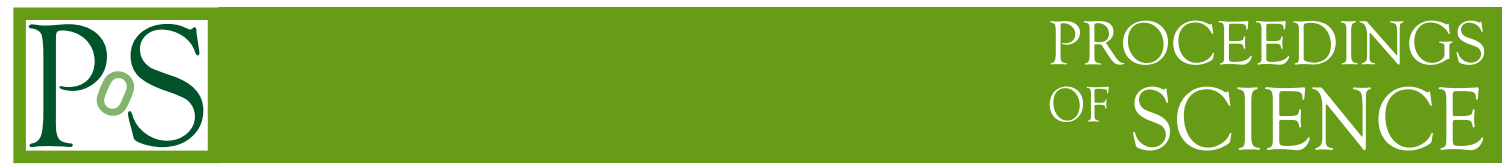

\title{
Matching NLO QCD to Monte Carlo showers and POWHEG
}

\author{
Simone Alioli*i \\ LBNL \& UC Berkeley \\ E-mail: salioli@lbl.gov
}

\begin{abstract}
Recent developments and applications of the POWHEG method, to merge NLO calculations with Shower Monte Carlo programs in the POWHEG-BOX framework, are presented. Particular emphasis is given to new improvements and automation and to the implementations of Dijets and top-quark pairs in association with a hard jet.
\end{abstract}

Loops and Legs in Quantum Field Theory - 11th DESY Workshop on Elementary Particle Physics, April 15-20, 2012

Wernigerode, Germany

\footnotetext{
*Speaker.

${ }^{\dagger}$ Based on works done in collaboration with J. Andersen, K, Hamilton, S. Moch, P. Nason, C. Oleari, E. Re, J. Smillie and P. Uwer
} 


\section{The POWHEG method}

Shower Monte Carlo event generators and NLO QCD calculations are important to give reliable predictions for signals and backgrounds relevant for collider phenomenology. To fully exploit the advantages of both the approaches it is clear that a method to include NLO QCD corrections to event generators is crucial, especially in view of the amount of data that LHC is collecting.

A merging method, that avoids the double counting of emissions, has been pioneered by MC@NLO [1] and later on followed by POWHEG [2,3]. The two approaches combine the accuracy of exact hard matrix elements for the large angle scattering - including all the radiative corrections to first order in the strong coupling constant $\alpha_{s}$ - with the soft and collinear emission described by the parton shower. In this way, they achieve both a reduced sensitivity with respect to variations of the unphysical renormalization and factorization scales, the correct Sudakov suppression of collinear and soft emissions and, basing on phenomenological models of hadrons formation, they produce realistic events with the same final state particles that can be observed in data. At variance with MC@NLO, the POWHEG method allows for the generation of positive weighted events only and is also independent of the parton SMC generator used. This independence is achieved by generating the hardest radiation, i.e. that with the highest transverse momentum, first and then letting the SMC perform the remaining shower, but requiring subsequent emissions not to be harder than the first. Mathematically, this corresponds to generate the hardest emission according to

$$
d \sigma=\bar{B}\left(\Phi_{B}\right) d \Phi_{B}\left[\Delta_{R}\left(p_{T}^{\min }\right)+\frac{R\left(\Phi_{R}\right)}{B\left(\Phi_{B}\right)} \Delta_{R}\left(k_{T}\left(\Phi_{R}\right)\right) d \Phi_{\mathrm{rad}}\right]
$$

where $B\left(\Phi_{B}\right)$ is the leading order contribution,

$$
\bar{B}\left(\Phi_{B}\right)=B\left(\Phi_{B}\right)+\left[V\left(\Phi_{B}\right)+\int d \Phi_{\mathrm{rad}} R\left(\Phi_{R}\right)\right]
$$

is the NLO differential cross section integrated on the radiation variables while keeping the Born kinematics fixed $\left(V\left(\Phi_{B}\right)\right.$ and $R\left(\Phi_{R}\right)$ stand respectively for the virtual and the real corrections to the Born process), and

$$
\Delta_{R}\left(p_{T}\right)=\exp \left[-\int d \Phi_{\operatorname{rad}} \frac{R\left(\Phi_{R}\right)}{B\left(\Phi_{B}\right)} \theta\left(k_{T}\left(\Phi_{R}\right)-p_{T}\right)\right]
$$

is the POWHEG Sudakov. With $k_{T}\left(\Phi_{R}\right)$ we denote the transverse momentum of the emitted particle. The cancellation of soft and collinear singularities is understood in the expression within the square bracket in eq. (1.2). Partonic events with hardest emission generated according to eq. (1.1) are then showered with a $k_{T}$-vetoed shower ${ }^{1}$.

Eq. (1.1) is useful to understand easily the main properties of the method. However, some amount of additional work is usually required to build a code that actually implements the features of eq. (1.1). For this reason, an automatic tool, dubbed POWHEG BOX [4], has been made available to ease the POWHEG implementation of new processes. It only requires as input the individual components of the NLO calculation under consideration, i.e., the Born process, its virtual radiative corrections and the real emission contributions. Then it automatically combines them, canceling the

\footnotetext{
${ }^{1}$ We refer the reader to the papers [2,3] where the details of the interface to the shower are properly discussed.
} 

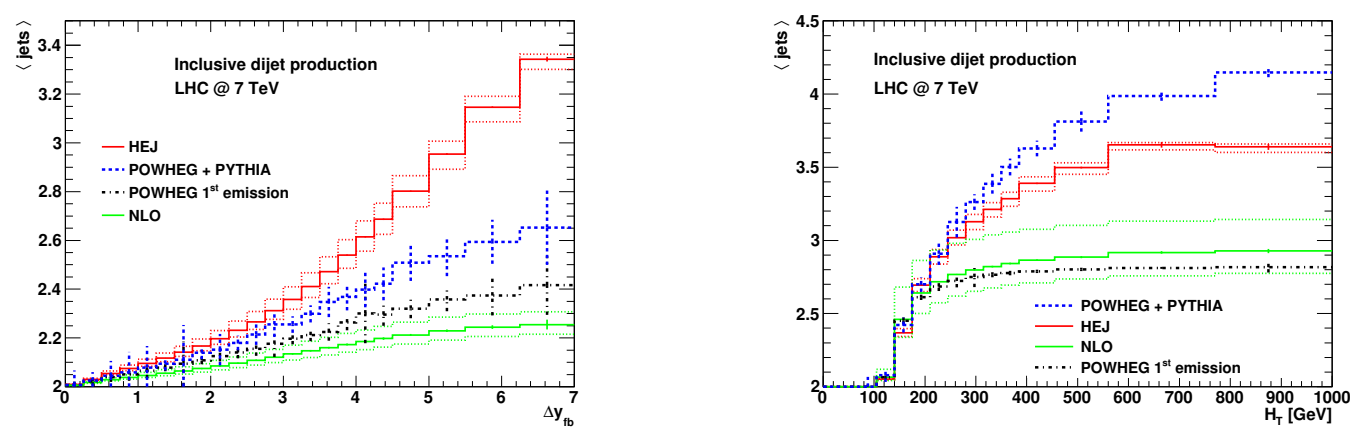

Figure 1: The average number of jets as a function of $\Delta y_{\mathrm{fb}}$ (left) and of $H_{\mathrm{T}}$ (right), as predicted by a fixed NLO calculation, by POWHEG first emission, by POWHEG+PYTHIA and by HEJ. The dotted red lines around the HEJprediction and the green ones around the NLO result are obtained by varying the renormalization and factorization scales by a factor of 2 around their central value.

emerging soft and collinear singularities in the Frixione-Kunszt-Signer (FKS) subtraction scheme, and produce the required events. The POWHEG BOX may also be seen as a library, where previously implemented processes are available in a common framework. Recently the automatic generation of the Born process, together with the color and helicity-correlated Born amplitudes needed to construct the subtraction counter-terms, has been added to the POWHEG BOX package. It relies on a modification of MADGRAPH, presented in [5]. On the same grounds, the interfacing to the automatic generation of virtual corrections via GOSAM [6] is currently being worked on, in order to fully automate the inclusion of a new process into the POWHEG BOX framework.

Recent implementations, which make use of some of the aforementioned additions, include jet pair production [7], $W^{+} W^{+}$plus dijet productions [8, 9], diboson [10], $W b \bar{b}$ [11] and Higgs plus up to 2 jets $[12,5]$ production. For an up-to-date list of included processes and in order to obtain the publicly available code, visit http://powhegbox.mib.infn.it/ .

\section{Higher order effects in Dijet production}

Recently both the ATLAS and CMS collaborations have sought for effects beyond pure nextto-leading order in dijet observables, aiming to understand whether a framework based on a (possibly next-to-leading-order-matched) parton shower (which resums the radiation resulting from a large ratio in transverse scales) is sufficient for the description of additional jets, or whether BFKLtype effects from hard, wide-angle emissions, have already become important at the center of mass energy of the LHC (7 TeV in the present study). Results presented in their studies are not yet conclusive.

We have performed a comparative study [13], identifying the regions of phase space in dijet production where some observables receive large corrections beyond next-to-leading order and studied their theoretical description with two tools that perform these two different resummations: the POWHEG BOX and HEJ [14-16]. We have also suggested analyses where the predictions from POWHEG and HEJ can be clearly distinguished experimentally. 


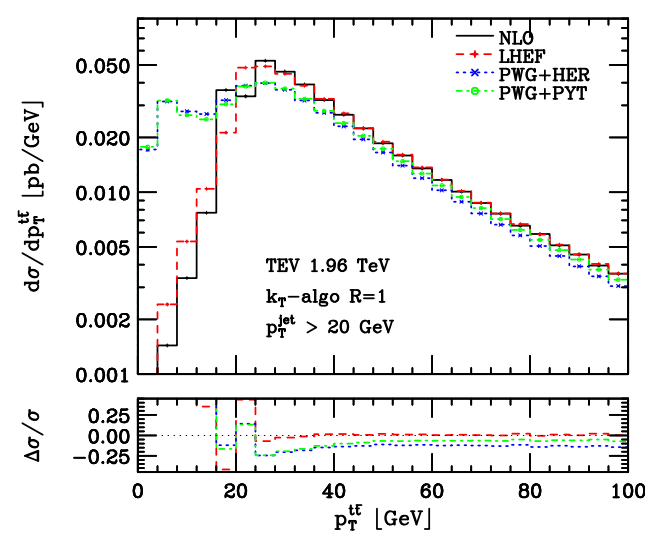

Figure 2: Differential cross section as a function of the $t \bar{t}$-pair transverse momentum at the Tevatron $(\sqrt{s}=1.96 \mathrm{TeV})$.

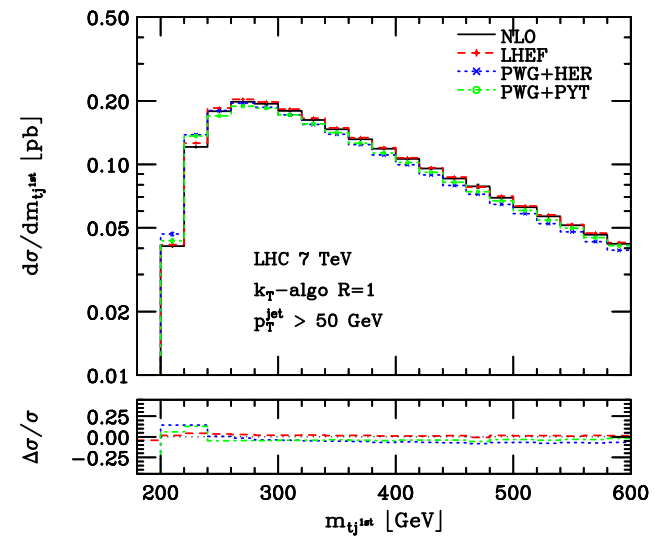

Figure 3: Differential cross section as a function of the $\left(t j_{1}\right)$ invariant mass at the $\operatorname{LHC}(\sqrt{s}=$ $7 \mathrm{TeV})$.

In Fig. 1, we plot on the left the average number of jets as a function of the rapidity difference $\Delta y_{\mathrm{fb}}$ between the most forward and most backward of the jets fulfilling

$$
p_{\mathrm{T}}^{j}>35 \mathrm{GeV}, \quad p_{\mathrm{T}}^{j_{1}}>45 \mathrm{GeV}, \quad\left|y_{j}\right|<4.7,
$$

and the same quantity as a function of $H_{\mathrm{T}}=\sum_{j} p_{\mathrm{T}}^{j}$ on the right. From the figures, it appears that greater distinguishing power between the different theoretical approaches can be achieved. In particular, the figure on the left shows that the wide-angle resummation implemented in HEJ produces more hard jets than POWHEG and the fixed NLO calculation, as $\Delta y_{\mathrm{fb}}$ increases. On the contrary, the average number of jets dependence with respect to $H_{\mathrm{T}}$ shows much larger values in the showered events.

\section{3. $t \bar{t}+$ jet hadroproduction}

In the following we concentrate on the recent implementation of the $t \bar{t}+$ jet hadroproduction in the POWHEG approach, presented in Ref. [17]. A large fraction of the inclusive $t \bar{t}$ production does indeed actually contain events with one or even more additional jets. Furthermore, due to the larger phase space available, the relative importance of data samples with $t \bar{t}+$ jets is larger at the LHC with respect to the Tevatron, increasing the need of an accurate theoretical description of this process. Top-quark pair-production associated with jets is also an important background to Higgs boson production in vector boson fusion and for many signals of new physics. The implementation reported here is based on the NLO QCD corrections evaluated in Ref. [18, 19], merged with HERWIG [20] and PYTHIA [21] SMC programs, using the POWHEG BOX.

We present results for both Tevatron and LHC colliders, having assumed a jet reconstruction cut in the analysis of $p_{\mathrm{T}}>20 \mathrm{GeV}$ and $50 \mathrm{GeV}$, respectively. We have used the inclusive- $k_{T}$ jet algorithm with $R=1$ and the $E_{T}$-recombination scheme. Renormalization and factorization scales have been set to $\mu_{R}=\mu_{F}=m_{t}=174 \mathrm{GeV}$, we have used the PDF set CTEQ6M, and we have not imposed any extra acceptance cut, other than those necessary to define the hard jet. 


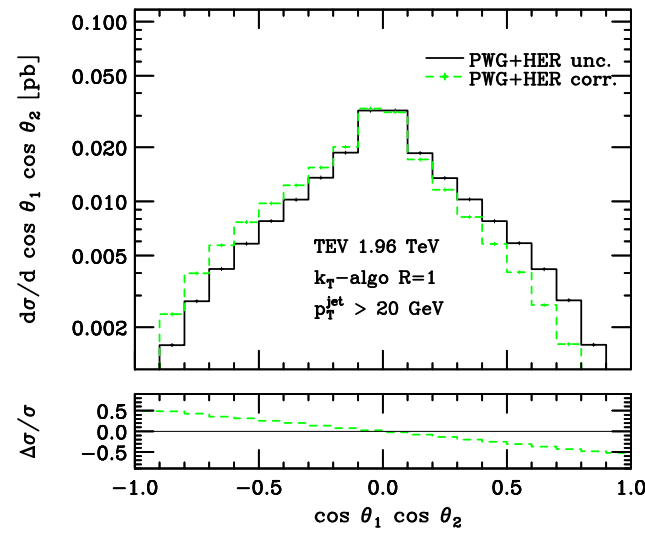

Figure 4: Effect of the inclusion of spin correlations when interfacing to HERWIG.

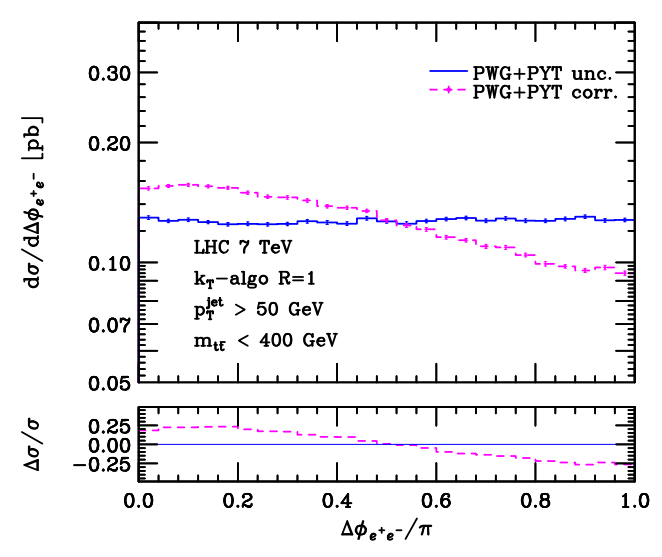

Figure 5: Effect of the inclusion of spin correlations when interfacing to PYTHIA.

In Fig. 2 we show the differential cross section as a function of the transverse momentum of the $t \bar{t}$-pair at the Tevatron, while in Fig. 3 we plot the invariant mass of the system made by the top-quark and the hardest jet at the $7 \mathrm{TeV}$ LHC. The different curves appearing on each plot refer respectively to the fixed order results (NLO), to the results after the first emission has been performed by POWHEG (LHEF) and to the fully showered events, with HERWIG (PWG+HER) Or PYTHIA $(\mathrm{PWG}+\mathrm{PYT})$ showers. Shower effects are visible in the low- $p_{\mathrm{T}}^{t \bar{t}}$ region, while more inclusive observables like the invariant mass of the system made by the top-quark and the hardest jet, $m_{\left(t j_{1}\right)}$, are basically unaffected by the shower.

In our implementation we have also included the spin-correlations between the production and decay stages. In doing so, we have neglected off-shell effects and non-resonant production mechanisms. We proceeded by first generating events with stable top-quarks (un-decayed events) through the usual POWHEG machinery and then generating the decay products according to the matrix element for the full production and decay process (decayed events), following Ref. [22]. In our study we always assumed the semi-leptonic top-quark decay channel $t \rightarrow W^{+} b \rightarrow \ell^{+} v b$. In Fig. 4 we draw the differential distribution $\frac{1}{\sigma} \frac{d^{2} \sigma}{d \cos \theta_{1} d \cos \theta_{2}}$ after the HERWIG shower, at the Tevatron collider, with $\theta_{1}$ and $\theta_{2}$ being the angles between the directions of the flights of the leptons coming from the decayed top-quark in the $t(\bar{t})$ rest frame and the beam axis, whose direction defines the quantization axis for the (anti-)top-quark spin. No extra acceptance cut is imposed on the leptons. In Fig. 5 we show instead the differential cross section as a function of the azimuthal distance between the two leptons coming from the top-quarks decays, for the LHC collider configuration and after the PYTHIA shower. An extra cut $m_{t \bar{t}}<400 \mathrm{GeV}$ has been imposed here to enhance the effect. Looking at both plots, it is possible to appreciate the differences between spin-correlated results and those obtained by letting the respective SMC program perform the uncorrelated top-quark decays.

We have also investigated the $t \bar{t}$ charge asymmetry in presence of a hard jet, finding that the inclusion of the shower changes significantly the fixed-order predictions in the low $p_{T}^{t \bar{t}}$ region, where shower effects are known to be large. Away from this region the parton shower leads only to a marginal change of the charge asymmetry binned in $p_{T}^{t \bar{t}}$. This quantity is now available at NLO accuracy, supplemented by the shower. For more details and for complete tables including results obtained with different cuts and at various stages of the simulation, we refer to Ref. [17]. 


\section{References}

[1] S. Frixione and B. R. Webber, JHEP 0206 (2002) 029 [arXiv:hep-ph/0204244].

[2] P. Nason, JHEP 0411 (2004) 040 [arXiv:hep-ph/0409146].

[3] S. Frixione, P. Nason and C. Oleari, JHEP 0711 (2007) 070 [arXiv:0709.2092 [hep-ph]].

[4] S. Alioli, P. Nason, C. Oleari and E. Re, JHEP 1006 (2010) 043 [arXiv:1002.2581 [hep-ph]].

[5] J. M. Campbell, R. K. Ellis, R. Frederix, P. Nason, C. Oleari and C. Williams, JHEP 1207 (2012) 092 [arXiv:1202.5475 [hep-ph]].

[6] G. Cullen, N. Greiner, G. Heinrich, G. Luisoni, P. Mastrolia, G. Ossola, T. Reiter and F. Tramontano, Eur. Phys. J. C 72, 1889 (2012) [arXiv:1111.2034 [hep-ph]].

[7] S. Alioli, K. Hamilton, P. Nason, C. Oleari, E. Re, JHEP 1104 (2011) 081. [arXiv:1012.3380 [hep-ph]].

[8] T. Melia, P. Nason, R. Rontsch, G. Zanderighi, Eur. Phys. J. C71 (2011) 1670. [arXiv:1102.4846 [hep-ph]]

[9] B. Jager, G. Zanderighi, [arXiv:1108.0864 [hep-ph]].

[10] T. Melia, P. Nason, R. Rontsch, G. Zanderighi, [arXiv:1107.5051 [hep-ph]].

[11] C. Oleari, L. Reina, JHEP 1108 (2011) 061. [arXiv:1105.4488 [hep-ph]].

[12] S. Alioli, P. Nason, C. Oleari and E. Re, JHEP 0904, 002 (2009) [arXiv:0812.0578 [hep-ph]].

[13] S. Alioli, J. R. Andersen, C. Oleari, E. Re and J. M. Smillie, Phys. Rev. D 85, 114034 (2012) [arXiv:1202.1475 [hep-ph]].

[14] J. R. Andersen and J. M. Smillie, JHEP 1001 (2010) 039 [arXiv:0908.2786 [hep-ph]].

[15] J. R. Andersen and J. M. Smillie, Phys. Rev. D 81, 114021 (2010) [arXiv:0910.5113 [hep-ph]].

[16] J. R. Andersen and J. M. Smillie, JHEP 1106, 010 (2011) [arXiv:1101.5394 [hep-ph]].

[17] S. Alioli, S. -O. Moch, P. Uwer, [arXiv:1110.5251 [hep-ph]].

[18] S. Dittmaier, P. Uwer, S. Weinzierl, Phys. Rev. Lett. 98 (2007) 262002. [hep-ph/0703120 [HEP-PH]].

[19] S. Dittmaier, P. Uwer, S. Weinzierl, Eur. Phys. J. C59 (2009) 625-646. [arXiv:0810.0452 [hep-ph]].

[20] G. Corcella et al., JHEP 0101 (2001) 010 [arXiv:hep-ph/0011363].

[21] T. Sjostrand, S. Mrenna and P. Z. Skands, JHEP 0605 (2006) 026 [arXiv:hep-ph/0603175].

[22] S. Frixione, E. Laenen, P. Motylinski, B. R. Webber, JHEP 0704 (2007) 081. [hep-ph/0702198 [HEP-PH]].

[23] S. Frixione, P. Nason and G. Ridolfi, JHEP 0709, 126 (2007) [arXiv:0707.3088 [hep-ph]].

[24] W. Bernreuther, J. Phys. G 35, 083001 (2008) [arXiv:0805.1333 [hep-ph]].

[25] S. Frixione, E. Laenen, P. Motylinski and B. R. Webber, JHEP 0603, 092 (2006) [arXiv:hep-ph/0512250].

[26] S. Frixione, E. Laenen, P. Motylinski, B. R. Webber and C. D. White, JHEP 0807, 029 (2008) [arXiv:0805.3067 [hep-ph]].

[27] G. Mahlon and S. J. Parke, Phys. Lett. B 476, 323 (2000) [arXiv:hep-ph/9912458]. 